\title{
CAPACIDADE DE ENRAIZAMENTO DE VARIEDADES DE NESPEREIRA SUBMETIDAS À PODA DE RENOVAÇÃO'
}

\author{
ERIVALDO JOSÉ SCALOPPI JUNIOR ${ }^{2}$, NATANAEL DE JESUS ${ }^{3}$, ANTONIO BALDO GERALDO MARTINS ${ }^{4}$
}

\begin{abstract}
RESUMO - O presente trabalho avaliou a capacidade de enraizamento de cinco variedades de nespereira (Eriobotrya japonica Lindl.), sendo Champagne, Precoce de Itaquera, Mizuho, Mogui e Tanaka, obtidas pela poda de renovação. Estacas tenras apicais, contendo um par de folhas e cerca de $15 \mathrm{~cm}$, foram tratadas por cinco segundos com ácido indolbutírico (IBA) $\left(0 ; 1.000 ; 3.000 ; 5.000\right.$ e $\left.7.000 \mathrm{mg} . \mathrm{L}^{-1}\right)$. O experimento foi conduzido em câmara de nebulização intermitente pertencente à UNESP/FCAV (21 $\left.15^{\prime} 22^{\prime \prime} \mathrm{Se} 48^{\circ} 18^{\prime} 58^{\prime \prime} \mathrm{W}\right)$. O delineamento experimental foi inteiramente casualizado, em esquema fatorial 5x5 (5 variedades e 5 concentrações de IBA), com 4 repetições e 10 estacas por parcela. As avaliações foram feitas após 90 dias. Os resultados indicaram um incremento de enraizamento e número de raízes crescente com a concentração de IBA utilizada, com exceção da variedade Mogui, que apresentou melhores resultados apenas quanto ao comprimento de raízes. Posterior ao transplantio, a sobrevivência das mudas foi alta (90\%), apresentando condições de serem plantadas no campo, aos seis meses, ou de serem enxertadas aos oito meses, decorrido desde o estaqueamento. Termos para indexação: Eriobotrya japonica, propagação vegetativa, estaquia, IBA, poda.
\end{abstract}

\section{ROOTING CAPACITY OF VARIETIES OF LOQUAT}

ABSTRACT - This work aimed to study the rooting capacity of five varieties of loquat (Eriobotrya japonica Lindl.), Champagne, Precoce de Itaquera, Mizuho, Mogui and Tanaka. Plants of 20 years of age were submitted to severely pruning. After budding apical softwood cuttings were taken and treated (5 seconds) with concentrations of indolbutiric acid (IBA) $\left(0,1000,3000,5000\right.$ and $\left.7000 \mathrm{mg}^{-\mathrm{L}^{-1}}\right)$. The cuttings were placed in drilled box supplied with vermiculite under intermitent mist inside a lathhouse belonging to UNESP/FCAV ( $\left.21^{\circ} 15^{\prime} 22^{\prime \prime} \mathrm{S} \mathrm{e} 48^{\circ} 18^{\prime} 58^{\prime \prime} \mathrm{W}\right)$. The experimental design used was the complete randomized, factorial scheme $5 \times 5$ ( 5 epecies and 5 IBA concentrations), four repetitions with 10 cuttings per plot. The evaluations were made after 90 days. The results indicated an increment of rooting and number of roots with the increase of concentration of IBA, except for the variety Mogui that just presented better results for the length of roots. The cuttings presented high survival (90\%), with conditions to be planted in the field after the sixth month (from the cutting) or be grafted in the eighth month.

Index terms: Eriobotrya japonica, vegetative propagation, cutting, adventicious rooting, IBA.

\section{INTRODUÇÃO}

A nespereira (Eriobotrya japonica Lindl.) é uma árvore perenefólia da família Rosaceae, originária da China Oriental e Japão, em regiões subtropicais, sendo cultivada desde tempos remotos (Cavaco, 1990). A maturação dos frutos de nêspera ocorre de maio a outubro no Estado de São Paulo, período de maior escassez de fruta in natura no mercado. Este fato tem possibilitado lucros mais compensadores ao produtor, além do aspecto atraente do fruto, de sabor suave e agradável. As nêsperas prestam-se também à produção de excelentes geléias e compotas, atividades ainda pouco exploradas (Ojima et al., 1999).

As mudas de nêspera são normalmente obtidas através da enxertia. Em relação à sua formação, são utilizadas sementes para a produção de porta-enxertos, resultando em um stand não uniforme quanto ao vigor e constituição genética. A propagação vegetativa, via estaquia, tem por finalidade proporcionar uniformidade das plantas, além da redução do tempo de formação da muda (Hartmann et al., 1997). A propagação por estaquia pode ser influenciada por diversos fatores, entre características inerentes à própria planta e condições do meio ambiente (Calabrese, 1978; Menzel, 1985). Dentre os fatores que podem melhorar os resultados, destacam-se a presença de folhas na estaca, utilização de câmara com nebulização intermitente, reguladores de crescimento, estádio de desenvolvimento da planta e do ramo, além da época do ano em que as estacas são coletadas.

Em relação ao estádio de desenvolvimento da planta e do ramo, tem-se o conceito da juvenilidade, sendo a capacidade de iniciar, prontamente, raízes adventícias, uma das principais características fisiológicas que parece comum a todas as plantas (Janick, 1966). Em espécies de difícil enraizamento, pode ser útil induzir, em plantas adultas, um rejuvenescimento para aumentar a capacidade de enraizamento (Hartmann et al., 1997).

O presente trabalho tem por objetivo avaliar a capacidade de enraizamento de cinco variedades de nespereira a serem propagadas por estaquia e do ácido indolbutírico (IBA) neste processo.

\section{MATERIAL E MÉTODOS}

Nespereiras obtidas através de enxertia, das variedades Champagne, Precoce de Itaquera, Mizuho, Mogui e Tanaka, com cerca de 15 anos de idade, provenientes do Câmpus da Unesp em Jaboticabal$\mathrm{SP}$, foram submetidas à poda de renovação de copa, deixando-se apenas as pernadas principais. Após a poda, ramos não lignificados (tenros) foram formados, os quais serviram de material para a realização do experimento. Foram coletadas estacas apicais com cerca de $15 \mathrm{~cm}$, contendo um par de folhas no nó superior, eliminando-se as demais folhas e feito um corte em bisel na extremidade inferior. Após preparadas, as estacas foram tratadas por imersão rápida, durante cinco segundos em IBA, nas concentrações: 0 (testemunha); 1.000; 3.000; 5.000 e 7.000 $\mathrm{mg} . \mathrm{L}^{-1}$, sendo em seguida estaqueadas em bandejas plásticas perfuradas, preenchidas com vermiculita de grânulos médios. O experimento foi conduzido em câmara de nebulização intermitente, pertencente ao Departamento de Produção Vegetal da Unesp em Jaboticabal-SP; o sistema de nebulização era acionado por "timer" e programado para manter um filme d'água na superfície das folhas. Todo o ensaio foi conduzido sob condições de ripado, com $50 \%$ de luminosidade.

O experimento foi realizado em 08 de abril de 2002 e, após noventa dias, foram feitas avaliações das estacas em relação à sobrevivência, enraizamento, número e comprimento médio de raízes, estacas calejadas, calejamento associado à presença de raiz e permanência de folhas.

Posterior à avaliação das estacas, estas foram transferidas para sacos plásticos contendo uma mistura de solo + areia + esterco de curral curtido (3:3:1 v), comumente utilizado no viveiro de mudas desta instituição e avaliadas quanto a sua sobrevivência e desenvolvimento inicial.

O delineamento experimental foi inteiramente casualizado, em esquema fatorial $5 \times 5$, com 5 variedades e 5 concentrações de IBA,

\footnotetext{
${ }^{1}$ (Trabalho 118/2003). Recebido: 04/09/2003. Aceito para publicação: 20/02/2004.

${ }_{2}^{2}$ Pós-graduando em Agronomia, área de concentração em Produção Vegetal, Universidade Estadual Paulista, Faculdade de Ciências Agrárias e Veterinárias (UNESP/ FCAV), Câmpus de Jaboticabal. Via de acesso Prof. Paulo Donato Castellane s/n, CEP 14.884-900, Jaboticabal-SP. Tel.: 163209 2668. e-mail: sjunior@ fcav.unesp.br. ${ }_{3}^{3}$ Pós-graduando em Agronomia, Departamento Produção Vegetal, UNESP/FCAV. e-mail: natanael@ fcav.unesp.br.

${ }^{4}$ Prof. Dr., Departamento Produção Vegetal, UNESP/FCAV. e-mail: baldo@ @cav.unesp.br.
} 
TABELA 1 - Valores e significância de F para os fatores variedade, concentração e interação em relação à permanência de folhas, calejamento, calejamento associado à raiz, enraizamento, sobrevivência, número e comprimento de raízes. Jaboticabal, UNESP/FCAV, 2003.

\begin{tabular}{|c|c|c|c|c|c|c|c|}
\hline Causas de variação & $\begin{array}{l}\text { Permanência } \\
\text { folhas }\end{array}$ & Calejamento & $\begin{array}{c}\text { Calejamento } \\
+ \text { raiz }\end{array}$ & Enraizamento & Sobrevivência & $\begin{array}{l}\text { Número } \\
\text { raízes }\end{array}$ & $\begin{array}{c}\text { Comprimento } \\
\text { raízes }\end{array}$ \\
\hline Variedade & $5,1^{* *}$ & $2,8^{*}$ & $1,6^{\mathrm{ns}}$ & $2,6^{*}$ & $4,8^{* *}$ & $3,1^{*}$ & $1,3^{\mathrm{ns}}$ \\
\hline Concentração & $0,7^{\mathrm{ns}}$ & $0,2^{\mathrm{ns}}$ & $0,2^{\mathrm{ns}}$ & $10,0 * *$ & $0,6^{\mathrm{ns}}$ & $15,1^{* *}$ & $5,5^{* *}$ \\
\hline Variedade $\mathrm{x}$ concentração & $0,8^{\mathrm{ns}}$ & $0,7^{\mathrm{ns}}$ & $1,0^{\mathrm{ns}}$ & $1,1^{\mathrm{ns}}$ & $0,8^{\mathrm{ns}}$ & $1,0^{\mathrm{ns}}$ & $1,5^{\mathrm{ns}}$ \\
\hline C.V. $(\%)$ & 20,1 & 79,8 & 122,4 & 25,9 & 19,5 & 17,7 & 19,0 \\
\hline
\end{tabular}

${ }^{\mathrm{ns}}$ Não significativo. $*$ Significativo $(\mathrm{p}<0,05) . * *$ Significativo $(\mathrm{p}<0,01)$.

TABELA 2 - Médias das variedades de nespereira em relação à permanência de folhas, calejamento, enraizamento, sobrevivência e número de raízes. Jaboticabal, UNESP/FCAV, 2003.

\begin{tabular}{|c|c|c|c|c|c|}
\hline Variedades & Permanência folhas ${ }^{2}$ & Calejamento $^{2}$ & Enraizamento $^{2}$ & Sobrevivência $^{2}$ & Número raízes $^{3}$ \\
\hline Champagne & $63,3 b^{1}$ & $10,2 \mathrm{~b}$ & $50,6 \mathrm{ab}$ & $63,6 \mathrm{~b}$ & $1,7 \mathrm{a}$ \\
\hline Mizuho & $75,0 \mathrm{ab}$ & $18,8 \mathrm{ab}$ & $51,9 \mathrm{ab}$ & $75,0 \mathrm{ab}$ & $1,9 \mathrm{a}$ \\
\hline Precoce de Itaquera & $80,5 \mathrm{a}$ & $14,9 \mathrm{ab}$ & $55,2 \mathrm{a}$ & $80,5 \mathrm{a}$ & $1,8 \mathrm{a}$ \\
\hline Mogui & $70,6 \mathrm{ab}$ & 23,6 a & $42,9 \mathrm{~b}$ & $73,4 \mathrm{ab}$ & $1,7 \mathrm{a}$ \\
\hline Tanaka & $81,5 \mathrm{a}$ & $21,4 \mathrm{ab}$ & $53,9 \mathrm{ab}$ & $81,5 \mathrm{a}$ & $1,9 \mathrm{a}$ \\
\hline D.M.S. (Tukey) & 13,2 & 12,6 & 11,6 & 12,9 & 0,3 \\
\hline
\end{tabular}

${ }^{1}$ Médias na coluna, seguidas de mesma letra, não diferem entre si, pelo teste de Tukey $(\mathrm{p}>0,05)$.

${ }^{2}$ Dados transformados em arco-seno $(\mathrm{x}+0,5 / 100)$.

${ }^{3}$ Dados transformados em $\quad(x+0,5)$.

totalizando 25 tratamentos. Cada tratamento constou de 4 repetições, sendo 10 estacas por parcela. Os resultados foram submetidos à análise de variância pelo teste $\mathrm{F}$, ao teste de Tukey e à regressão polinomial. Para efeito da análise estatística, os dados em porcentagem relativos ao enraizamento, sobrevivência, calejamento, calejamento associado à raiz e permanência de folhas foram transformados em arco-seno $\sqrt{ }(x+0,5) /$ 100 , enquanto os valores referentes ao número e comprimento de raízes foram transformados em $\sqrt{ }(x+0,5)$

\section{RESULTADOS E DISCUSSÃO}

A Tabela 1 apresenta os valores e significância do teste $\mathrm{F}$ para os fatores variedade, concentração e interação em relação às características avaliadas. Com relação à permanência de folhas, $\mathrm{o}$ teste $\mathrm{F}$ apresentou significância apenas entre as variedades. A sobrevivência das estacas foi diferenciada entre as variedades, de forma semelhante à permanência de folhas. O calejamento foi diferenciado entre as variedades, não apresentando influência das concentrações do regulador utilizado, sendo um fato independente da indução radicular (Hartmann et al., 1997), o que pode ser constatado na associação da formação de calo e presença de raiz que não foi significante para variedade, concentração do regulador e interação. Normalmente, o aparecimento precoce de raízes pode prevenir o desenvolvimento do calo (Komissarov, 1968). O enraizamento foi significante ao nível de $5 \%$ para variedades e a $1 \%$ para as concentrações de IBA. O número de raízes foi significativo entre variedades e concentrações do regulador, à semelhança do enraizamento. Níveis de auxina (endógeno ou exógeno) são determinantes na diferenciação de muitos tipos de meristemas, incluindo raízes (Leopold, 1955). O comprimento de raízes foi significativo apenas para as concentrações de IBA.
A Tabela 2 apresenta as médias (valores transformados) das variedades de nespereira em relação à permanência de folhas, calejamento, enraizamento, sobrevivência e número de raízes. As variedades apresentaram comportamento semelhante para permanência de folhas e sobrevivência, em que as variedades Precoce de Itaquera e Tanaka diferiram da variedade Champagne, embora esta não tenha diferido das demais. Quanto ao enraizamento, as maiores médias são encontradas para Precoce de Itaquera, que diferiu apenas de Mogui. Observando-se as médias das variedades para permanência de folhas e sobrevivência, nota-se uma analogia nos resultados, sendo geralmente os maiores valores de uma variedade tendo correspondência entre as características e vice-versa; comprovando que a maior constância no suprimento de carboidratos, auxinas e outras substâncias promotoras produzidas pelas folhas pode influir no enraizamento e sobrevivência. Os valores encontrados para calejamento indicam diferença estatística apenas entre as variedades Mogui e Champagne, comprovando novamente a ocorrência independente entre calejamento e enraizamento (Hartmann et al., 1997), pois estas duas variedades apresentaram os menores valores de enraizamento. Embora o teste $\mathrm{F}$ indicasse significância para as variedades em relação ao número de raízes, o teste de Tukey não apresentou diferença estatística entre elas.

A Tabela 3 apresenta valores médios de enraizamento, número e comprimento de raízes das variedades de nespereira em função das concentrações de IBA. Em relação ao enraizamento, encontra-se uma relação direta de incremento, em que, a partir de $3.000 \mathrm{mg} . \mathrm{L}^{-1}$, houve diferença estatística no que se refere à testemunha, com tendência de estabilização dos valores a partir de $5.000 \mathrm{mg} . \mathrm{L}^{-1}$. De forma semelhante, verificam-se melhores resultados pelo uso do IBA para número e comprimento de raízes, ocorrendo estabilização dos valores a partir de $5.000 \mathrm{mg} . \mathrm{L}^{-1}$ e $3.000 \mathrm{mg} . \mathrm{L}^{-1}$, respectivamente, diferindo da testemunha.

TABELA 3 - Médias das concentrações de IBA em relação ao enraizamento, número e comprimento de raízes. Jaboticabal, UNESP/FCAV, 2003.

\begin{tabular}{ccrr}
\hline Concentração IBA $\left(\mathrm{mg} . \mathrm{L}^{-1}\right)$ & Enraizamento $^{2}(\%)$ & Número raízes $^{3}$ & Comprimento raízes $^{3}\left(\mathrm{~cm}^{3}\right)$ \\
\hline 0 & $39,7 \mathrm{c}^{1}$ & $1,5 \mathrm{~b}$ & $2,5 \mathrm{~b}$ \\
1.000 & $42,5 \mathrm{bc}$ & $1,6 \mathrm{~b}$ & $3,0 \mathrm{ab}$ \\
3.000 & $53,9 \mathrm{ab}$ & $1,8 \mathrm{~b}$ & $3,1 \mathrm{a}$ \\
5.000 & $57,4 \mathrm{a}$ & $2,1 \mathrm{a}$ & $3,2 \mathrm{a}$ \\
7.000 & $60,9 \mathrm{a}$ & $2,1 \mathrm{a}$ & $3,2 \mathrm{a}$ \\
\hline D.M.S. (Tukey) & 11,6 & 0,3 & 0,5 \\
\hline
\end{tabular}

\footnotetext{
${ }^{1}$ Médias na coluna, com mesma letra, não diferem entre si, pelo teste de Tukey $(\mathrm{p}>0,05)$.

${ }^{2}$ Dados transformados em arco-seno $(\mathrm{x}+0,5 / 100)$.

${ }^{3}$ Dados transformados em $\quad(x+0,5)$.
} 

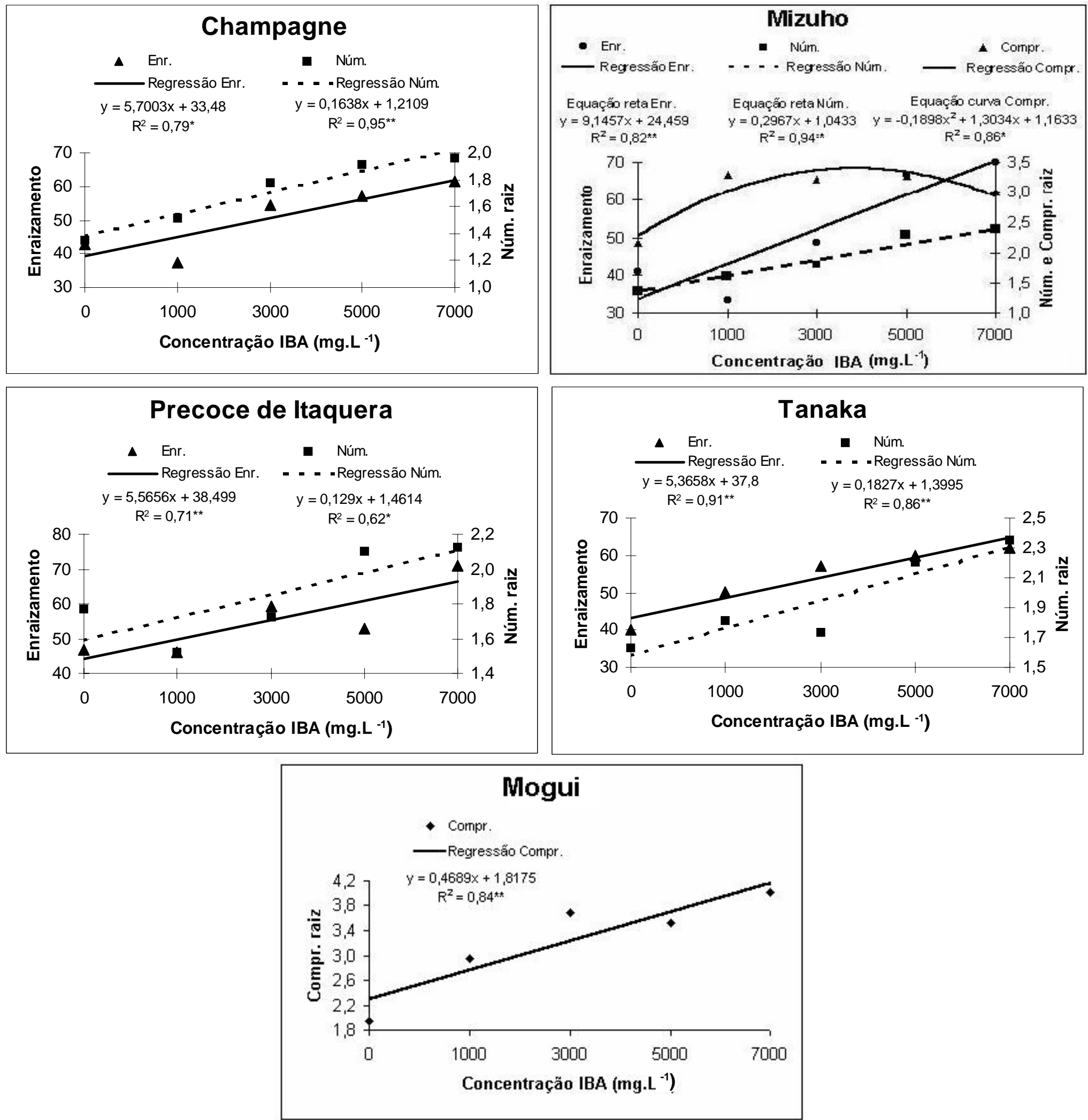

FIGURA 1 - Curvas de regressão (dados transformados) das variedades de nespereira entre as concentrações de IBA e as características avaliadas, sendo enraizamento (enr.), número (núm.) e comprimento de raízes (compr.). Jaboticabal, UNESP/FCAV, 2003.

A máxima eficiência técnica das concentrações de IBA pode ser observada na Figura 1 para cada variedade, em que são apresentadas as regressões polinomiais significantes para enraizamento, número e comprimento de raízes. Os pontos de máximo, obtidos pela derivação das equações, indicam no geral maiores valores na maior concentração de IBA (7.000 mg. $\left.\mathrm{L}^{-1}\right)$; com exceção para a curva de regressão do comprimento de raízes na variedade Mizuho, em que o ponto de máximo se situa entre as concentrações de 3.000 e $5.000 \mathrm{mg} . \mathrm{L}^{-1}$. Todas as variedades apresentaram incremento significativo de enraizamento pelo uso do IBA, com exceção da variedade Mogui, que apresentou significância apenas para o comprimento de raízes. As raízes possuem alta sensibilidade às auxinas, e o fornecimento exógeno pode prejudicar o crescimento destas (Leopold, 1955). O IBA promove apenas uma precocidade na formação de raízes (Pereira et al., 1991) e diferenciação dos primórdios radiculares existentes, além do estímulo na formação de novos primórdios (Komissarov, 1968). O maior comprimento de raízes nas variedades Mizuho e principalmente Mogui é devido, provavelmente, à precocidade na emissão de raízes estimulada pelo uso do IBA.

Pereira et al. (1996) realizaram estaquia de nespereira sem regulador de crescimento e verificaram que a variedade Mizuho enraíza melhor com estacas colhidas no verão do que no inverno. Estacas apicais apresentando meia-folha foram melhores do que estacas subapicais. $\mathrm{O}$ 
enraizamento esperado foi de 25 a $36 \%$. O presente experimento foi realizado no outono, sendo um período intermediário ao ótimo, segundo Pereira et al. (1996), porém a poda de rejuvenescimento permitiu resultados, mesmo da testemunha, superiores aos encontrados pelo autor citado (Tabela 4).

Boliani (1986) utilizou estacas herbáceas enfolhadas de nespereira das variedades Mizuho e Precoce de Itaquera, colhidas no

TABELA 4 - Médias de enraizamento (\%), número e comprimento $(\mathrm{cm})$ de raízes das variedades de nespereira em função das concentrações de IBA $\left(\mathrm{mg} . \mathrm{L}^{-1}\right)$. Jaboticabal, UNESP/FCAV, 2003.

\begin{tabular}{|c|c|c|c|c|}
\hline Variedades & $\begin{array}{c}\text { Concentração } \\
\text { IBA } \\
\left(\mathrm{mg} \cdot \mathrm{L}^{-1}\right)\end{array}$ & $\begin{array}{c}\text { Enraizamento } \\
(\%)\end{array}$ & $\begin{array}{l}\text { Número } \\
\text { raiz }\end{array}$ & $\begin{array}{l}\text { Comprimento } \\
\text { raiz }(\mathrm{cm})\end{array}$ \\
\hline \multirow[t]{5}{*}{ Champagne } & 0 & 45,1 & 1,3 & 9,2 \\
\hline & 1.000 & 36,5 & 1,8 & 7,7 \\
\hline & 3.000 & 65,1 & 2,7 & 8,9 \\
\hline & 5.000 & 68,3 & 3,2 & 10,1 \\
\hline & 7.000 & 71,0 & 3,5 & 8,9 \\
\hline \multirow[t]{5}{*}{ Mizuho } & 0 & 43,1 & 1,3 & 5,0 \\
\hline & 1.000 & 30,8 & 2,4 & 10,7 \\
\hline & 3.000 & 55,8 & 2,9 & 10,1 \\
\hline & 5.000 & 82,2 & 5,5 & 10,2 \\
\hline & 7.000 & 84,4 & 5,6 & 8,3 \\
\hline \multirow{5}{*}{$\begin{array}{l}\text { Precoce de } \\
\text { Itaquera }\end{array}$} & 0 & 52,5 & 2,7 & 7,7 \\
\hline & 1.000 & 51,1 & 1,8 & 9,4 \\
\hline & 3.000 & 71,9 & 2,5 & 8,4 \\
\hline & 5.000 & 61,4 & 4,2 & 9,3 \\
\hline & 7.000 & 85,0 & 4,1 & 10,2 \\
\hline \multirow[t]{5}{*}{ Mogui } & 0 & 26,4 & 1,8 & 4,1 \\
\hline & 1.000 & 50,6 & 1,9 & 8,2 \\
\hline & 3.000 & 56,7 & 2,7 & 13,2 \\
\hline & 5.000 & 58,6 & 3,3 & 11,9 \\
\hline & 7.000 & 42,5 & 2,4 & 16,1 \\
\hline \multirow[t]{5}{*}{ Tanaka } & 0 & 41,4 & 2,2 & 6,3 \\
\hline & 1.000 & 58,1 & 2,8 & 7,6 \\
\hline & 3.000 & 69,4 & 2,6 & 6,6 \\
\hline & 5.000 & 74,2 & 4,4 & 9,0 \\
\hline & 7.000 & 77,1 & 5,1 & 8,0 \\
\hline
\end{tabular}

final de outubro e obtidas após podas drásticas realizadas nas plantasmatrizes, com a utilização de IBA $\left(0 ; 2.000\right.$ e 4.000 mg.L $\left.\mathrm{L}^{-1}\right)$, obtendo médias de enraizamento de 46,8 e $52,3 \%$, respectivamente. Para a variedade Precoce de Itaquera, houve aumento de enraizamento com a aplicação de IBA, não acontecendo o mesmo com a variedade Mizuho. Os resultados contrastam com o presente em relação à resposta das variedades, demonstrando a necessidade de experimentos regionais para a determinação de um protocolo.

Posterior ao enraizamento, as estacas enraizadas foram transferidas para sacos plásticos contendo o substrato já descrito, permanecendo por mais um mês sob nebulização, para assegurar o pegamento e, após, foram mantidas em telado com $50 \%$ de sombreamento, recebendo água duas vezes ao dia por microaspersão. A sobrevivência das mudas foi alta (cerca de $90 \%$ ) no primeiro mês e manteve-se até o final da avaliação, com as mudas apresentando cerca de $50 \mathrm{~cm}$ de altura com um ano de idade, desde a instalação. Aos seis meses de idade, as mudas já apresentavam condições de serem plantadas no campo e, aos oito meses, com cerca de $30 \mathrm{~cm}$ de altura, possuíam diâmetro suficiente para a realização da enxertia (equivalente a um lápis). Em condições normais de desenvolvimento, as mudas provenientes de sementes estão aptas para receber a enxertia entre 15 e 18 meses após a semeadura, com altura aproximada de $80 \mathrm{~cm}$ e diâmetro de $1 \mathrm{~cm}$ (Ojima et al., 1999). Os resultados indicam precocidade na formação das mudas por estaquia.

\section{CONCLUSÕES}

O IBA promoveu incremento de enraizamento e no número de raízes, com exceção para a variedade Mogui. As estacas apicais tenras, provenientes da poda de renovação, apresentaram melhores resultados que nos experimentos encontrados na literatura. A sobrevivência e o estabelecimento das mudas foi alto, com precocidade em sua formação.

\section{REFERÊNCIASBIBLIOGRÁFICAS}

BOLIANI, A.C. Efeitos do estiolamento basal, da juvenilidade e do uso de um regulador vegetal no enraizamento de estacas de raízes e de ramos herbáceos de algumas espécies frutíferas. 1986. 129f. Dissertação (Mestrado em Fitotecnia) - Escola Superior de Agricultura "Luiz de Queiroz", Universidade de São Paulo, Piracicaba, 1986.

CALABRESE, F. Frutticoltura tropicale e subtropicale. Bologna: Coop. Lib. Un. Editrice, 1978. 498p.

CAVACO, A.L.P. A nespereira. In: PORTUGAL. Ministério da Agricultura, Secretaria de Estado da Agricultura, Direcção Regional de Agricultura do Algarve. FRUTICULTURA: experimentação frutícola no Algarve, alguns aspectos. Algarve: DRAAG/DDI, 1990. p.107-122.

HARTMANN, H,T.; KESTER, D.E.; DAVIES, F.T. Plant propagation: principles and practices. 6.ed. New Jersey: Prentice Hall, 1997. p.276501.

JANICK, J. A Ciência da horticultura. Rio de Janeiro: Freitas Bastos, 1966. 485p.

KOMISSAROV, D.A. Biological basics for the propagation of wood plants by cuttings. Jerusalem: IPST Press, 1968. 250p.

LEOPOLD, A.C. Auxins and plant growth. University California Press, Los Angeles. 1955, 354p.

MENZEL, C.M. Propagation of lychee: a review. Scientia Horticulturae, Amsterdam, v.25, n.1, p. 31-48, 1985.

OJIMA, M.; CAMPO DALL'ORTO, F.A.; BARBOSA, W.; MARTINS, F.P; SANTOS, R.R. Cultura da nespereira. Campinas: Instituto Agronômico, 1999. 36p. (Boletim técnico, 185).

PEREIRA, F.M.; PETRECHEN, E.H.; BENINCASA, M.M.P.; BANZATO, D.A. Efeito do ácido indolbutírico no enraizamento de estacas herbáceas de goiabeira (Psidium guajava L.) das cultivares Rica e Paluma, em câmara de nebulização. Científica, Jaboticabal, v.19, n.2, p.199-206, 1991

PEREIRA, F.M.; ZANIN, E.S.; BARBOSA, J.C. Efficiency of different treatments on the rooting of cuttings of the loquat (Eriobotrya japonica Lindl.) in a mist chamber. In: REUNIÃO INTERAMERICANADE HORTICULTURATROPICAL, 42., 1996, Curitiba, PR. Resumos... Londrina: IAPAR, 1996. p.490. 